\title{
000. HEILMITTEL GEGEN BLUTUNGEN AUS DEM UNTERLEIB, GEGEN SCHWELLUNGEN UND ANGSTZUSTÄNDE
}

Inv. 1850

10./11. Jh.
$16,5 \times 11 \mathrm{~cm}$

Tafel
Papier

Herkunft unbekannt

Auf diesem Stück Papier sind Heilmittel gegen drei unterschiedliche Leiden erhalten. Das Blatt ist dazu in drei verschiedene Abschnitte gegliedert worden, die durch Markierungslinien deutlich voneinander getrennt sind. Den Abschnitten sind jeweils eigene Zauberzeichen oder Zauberbuchstaben zugeordnet. Vermutlich war das Blatt einst wesentlich größer, denn dem linken unteren Abschnitt fehlen nicht nur die entsprechenden Zauberzeichen, sondern auch die Nennung des geeigneten Heilmittels selbst, das bei einer Schwellung zur Anwendung kommen sollte. Es könnte gar ein Drittel des unteren Blattes fehlen, denn die noch erhaltene horizontale Faltung befindet sich direkt in der Blattmitte. Wäre also der untere Blattrand verloren, müsste das Blatt einst horizontal zweimal gefaltet gewesen sein. Vertikal hingegen lassen sich sechs Faltungen erkennen.

Auf der Rückseite des Blattes findet sich, um $90^{\circ}$ nach rechts gedreht,

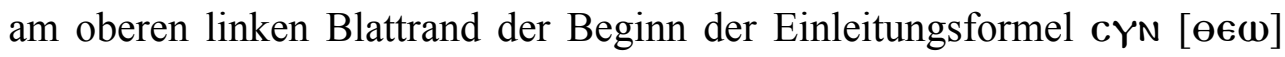
„Mit [Gott].“ Von einer weiteren Beschriftung ist anschließend jedoch Abstand genommen worden. Die Schrift der Vorderseite zeigt Charakteristika der Buchschrift des 10./11. Jh.s., ${ }^{1}$ wie etwa die linke Schlaufe des Lambdas und den linken Anstrich des Taus.

Die einzelnen Angaben $\mathrm{zu}$ den hier erhaltenen Heilmitteln sind vermutlich aus einer größeren Heilmittelsammlung zur akuten Behandlung kopiert worden. In der für Heilmittelsammlungen typischen Form werden sie mit der Präposition єTBє-, gelegentlich auch €-, ,,bezüglich“, gefolgt von einem Krankheitsbild, eingeleitet. Den jeweiligen Symptomen entsprechend werden die Möglichkeiten und Wege zur Linderung aufgeführt. In vielen solchen Rezeptsammlungen treten dabei auch vereinzelt Rezepte zur Frauenheilkunde in Erscheinung, die besonders häufig den Blut- oder Milchfluß

1 Siehe dazu die Bemerkungen und Schriftbeispiele bei V. Stegemann, Koptische Paläographie, Heidelberg 1936, S. 21f., Taf. 19 und 21, bes. 21.1 und 21.5. 
betreffen, ${ }^{2}$ und Hilfsmittel bei der Geburt eines Kindes aufführen. ${ }^{3}$ Auch im hier vorliegenden Kölner Fragment geht es zunächst um Blutungen aus dem weiblichen Unterleib, die es zu behandeln gilt. Dabei scheint es sich nicht um Schmerzlinderung bei verstärkter Regelblutung zu handeln, sondern um bedrohlichere bzw. verletzungsbedingte Blutungen, wie sie etwa während der Schwangerschaft oder nach der Geburt auftreten können. Einige solcher Heilmittel gegen krankhaften Blutfluß nach der Entbindung scheinen größtenteils aus Räuchermitteln bestanden zu haben. ${ }^{4}$ Auch gegen die im hier vorliegenden Papierfragment genannten Blutungen wird ein Doppelmittel vorgeschlagen, das zum einen aus einer, vermutlich vaginal angewendeten, Räucherung, zum anderen aus einem zu fertigenden Amulett bestand. Letzteres soll an den Unterleib der blutenden Frau geheftet werden. Die entsprechenden Zauberzeichen in ihrer vorgeschriebenen Anordnung, die mit Tinte auf Papyrus oder auf Holz geschrieben werden sollen, werden dazu direkt mitgeliefert. Um aus dieser Anleitung ein wirksames Heilmittel werden zu lassen, muss jedoch der Name der erkrankten Frau noch spezifiziert werden, denn in der letzten Zeile (12 links) ist nur vom Unterleib der

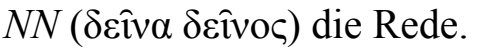

Gleiches gilt auch für das zweite Heilmittel (Zeile 3-16 rechts), das einer Person zugute kommen soll, deren Name an die Stelle des hier angegebenen $N N(\delta \varepsilon \hat{v} v \alpha \delta \varepsilon \hat{v}(\varsigma)$ einzusetzten ist. In diesem Fall handelt es sich jedoch um ein mentales Leiden, wie der Begriff $\mu \alpha v i ́ \alpha$ (Zeile 15 rechts) zeigt. Die Symptome dieses Leidens werden mit Angstzuständen und Weinen des Patienten umschrieben. Bei diesem handelt es sich um einen jungen Schreiber, Beamten oder Lehrer (cdxo), der sich seiner neuen Aufgabe nicht ganz gewachsen fühlt, oder vielelicht generell an einer Phobie leidet. Dass für

\footnotetext{
2 Siehe die Beispiele bei W. C. Till, Die Arzneikunde der Kopten, Akademie-Verlag Berlin 1951, S. 112, Rezept BA 2-11, und S. 113, Rezept BKU 5 und 9.

3 Siehe dazu zuletzt die Edition eines Papierfragments mit Heilmitteln bei K. Hevesi, The Coptic Medico-Magical Text K 11088 from the Papyus Collection of the Austrian National Library, in: Journal of Coptic Studies (JCoptS) 17 (2015), 55-83. Dort sollte mit Blick auf die Einführung des Rezepts A, Zeile 1-3 vermutlich €үсrıмє єc- „Bezüglich einer Frau, die ..." „Concerning a woman who ..." gelesen werden. Ebenso ist vermutlich

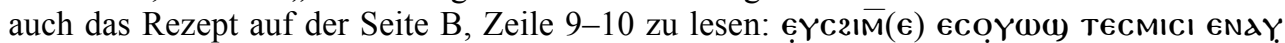
„Concerning a woman who wishes her offspring to come ...“ bzw. „Bezüglich einer Frau, die wünscht, dass es zur Geburt ihres Kindes kommt ..." Der feminine Possessivartikel TEC- statt des üblichen nєc- könnte sich dabei auf die Geburt eines weiblichen Kindes beziehen. NaY dort für NHY.
}

${ }^{4}$ A. Kropp, Ausgewählte koptische Zaubertexte, Bd. III, Brüssel 1930, 202. 
diesen Fall ein eigenes Schutzmittel bzw. festes Heilmittelschema existierte, deutet darauf hin, dass dieser Zustand häufiger auftrat.

Gegen Schwellungen, wie sie im letzten Abschnitt zur Sprache kommen, scheint dagegen ein dem König Salomon zugeschriebenes Heilmittel tradiert worden zu sein, dessen Details jedoch verloren sind. Der Grund für die Schwellung wird den Machenschaften eines Dämons zugeschrieben, der sich eines Körperteils des Patienten bemächtigt zu haben scheint. Eine solche Erklärung für krankhafte Schwellungen läßt sich auch in zahlreichen Sammlungen von Wunderheilungen durch Märtyrerheilige beobachten, wie etwa in den Miracula des heiligen Kolluthos, wo ein Dämon die Brüste einer Patientin befällt, die dadurch unheilbar anschwellen. ${ }^{5}$ Nur durch göttliche Hilfe und die Kraft eines Heiligen, oder hier des bekannten Dämonenbezwingers Salomon, ${ }^{6}$ lässt sich ein solcher Zustand heilen.

ETBE OYC2IME EPE OYCNOY 2APOC PNAI MOPOY EPOC

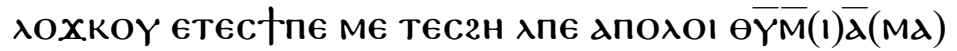

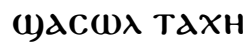

4 ZAUBERZEICHEN C2AI NAI NAגZAUBERZEICHEN MITET NAגOYPOEIC EYKOYI ZAUBERZEICHEN XOҮВI XАPcגхо EчPIME ZAUBERZEICHEN THC גoxkOY Ечнр2Оте •

8 ZAUBERZEICHEN ЄPOC OүCגI! пє ZAUBERZEICHEN ZAUBERZEICHEN EYTO KINWN ZAUBERZEICHEN ZAUBERZEICHEN OYKOIANA : $2 \overline{\mathrm{d}} \mathrm{N}-$ ZAUBERZEICHEN ZAUBERZEICHEN AMA2TE EXN TKZAUBERZEICHEN

12 ZAUBERZEICHEN $\boldsymbol{\lambda} \lambda \boldsymbol{\lambda} 2 \mathrm{H} \mathrm{N} \overline{\boldsymbol{\lambda}} \overline{\boldsymbol{\lambda}}$ ZAUBERZEICHEN ZAUBERZEICHEN TAגO MIETWWNE

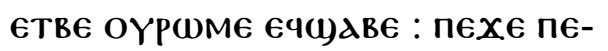
21 †2оте POEIC $\bar{\Delta} \bar{\lambda}$

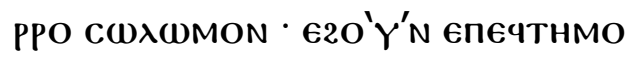
2M MIPIME MN TMANI'A':

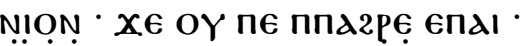
AlO AlO : TAXH TAXH

\footnotetext{
5 Siehe dazu zuletzt den Text dieser Wundergeschichte bei G. Schenke, Das koptisch hagiographische Dossier des Heiligen Kolluthos, Arzt, Märtyrer und Wunderheiler, CSCO 650, Leuven 2013, 216-217.

${ }^{6} \mathrm{Zu}$ Salomons Macht über Dämonen siehe den Aufsatz von P. Särkiö, Salomo und die Dämonen, Studia Orientalia 99, 2004, 305-322.
} 


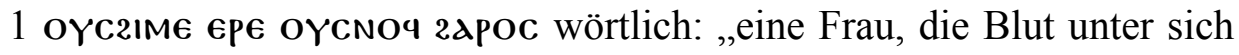
hat." Krankhafter Blutfluss wurde z.B. mit einem Homervers zu Apollon (Il. 1,75) als Amulett behandelt, wie PGM XXIIa, 2-9, zeigt, ließ sich bei Undankbarkeit nach der Heilung mit einem zweiten Vers (Il. 1,96) aber auch wieder entfachen. Amulette speziell gegen krankhaften Blutfluss bei Frauen finden sich in C. Bonner, Studies in Magical Amuletts, Ann Arbor 1950, 88-89 (Hinweis von R. Daniel). Auch im NT spielt bei der Heilung einer blutflüssigen Frau (Mt 9,20-22 und Mk 5,25-34) der feste Glaube an göttliche Kraft für die eigene Genesung eine wesentliche Rolle.

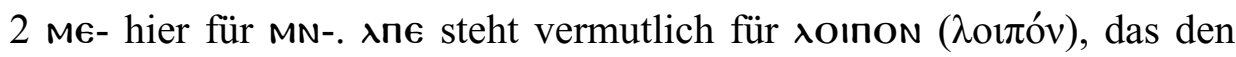
zweiten Teil des Rezepts einleitet. Das erste Omikron von anодо। (für $\dot{\alpha} \pi \circ \lambda \hat{v} \omega)$ ist in das Pi hinein geschrieben, das zweite dagegen über der Zeile geschrieben worden. Am Zeilenende scheint $\Theta \bar{\gamma} \bar{M}(1) \bar{\lambda}(M d)$ abgekürzt als $\Theta \bar{\gamma} \overline{M d}$ für $\theta v \mu i ́ \alpha \mu \alpha$ geschrieben zu stehen. $\mathrm{Zu}$ diesem Räucherwerk, das gelegentlich in Verbindung mit ả $\mu \mu \omega v \imath \alpha$ kóv als $\alpha \mu \mu \omega v \imath \alpha \kappa o ́ v ~ \theta v \mu i ́ \alpha \mu \alpha$, „Räucherammoniakum“, in Heilrezepturen Verwendung fand siehe Till, Arzneikunde, S. 47.5. Siehe ebenfalls dort das Rezept 5 auf S. 113, das für eine an Blutfluss leidende Frau ein Räucherheilmittel empfiehlt.

3 Der Aorist „sie pflegt sich schnell zu erholen“ hier vermutlich in futurischer Bedeutung.

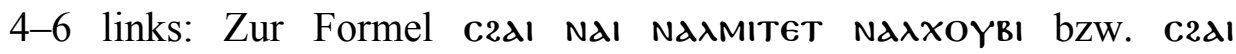

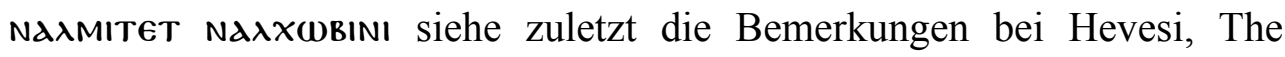
Coptic Medico-Magical Text K 11088, JCoptS 17, S. 74, Komm. z. Z. 5 , dort „Write with ink: Nalchobini.“ Stattdessen ist wohl in хоүв। eher das persische Wort choubi bzw. choob „Holz“ zu vermuten. Vielleicht ist in

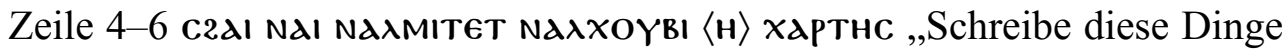
mit Tinte auf Holz 〈oder $\rangle$ Papyrus“" zu lesen.

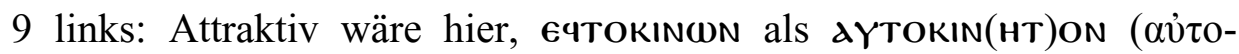

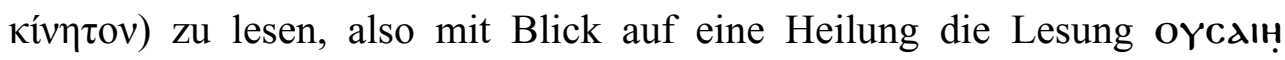
пєגYTOKIN(HT)ON „Schön ist das von selbst Verschwindende“ als Lesung $\mathrm{zu}$ erwägen, doch ist dafür von $\mathrm{zu}$ vielen Verschreibungen auszugehen. Näher liegt vermutlich die Lesung oүcalt nє єчто kINWN „Eine schöne Sache ist das, was gemeinsam (Kovvóv) angewendet ist", d.h. im hier vorliegenden Fall der Blutungen die vereinte Kraft von Zauberamulett und Räucherheilmittel zu verstehen. 
10 links: Bei oүkoidnd handelt es sich vermutlich um eine Mengenangabe für das zu verabreichende Räucherheilmittel, also oүkold für ở $\gamma$ -

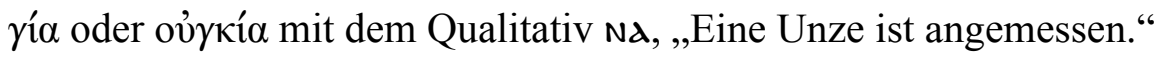

4-5 rechts: Die Bezeichnung caxo steht für ca2 o, also ,großer Schreiber" bzw. Berufsschreiber, Beamter, Notar oder Lehrer, siehe dazu den Eintrag bei Westendorf, Koptisches Handwörterbuch, 209. Der Zusatz oүкоү। deutet darauf hin, dass es sich hier um einen noch sehr jungen, angehenden oder zukünftigen professionellen Schreiber handeln könnte, unter Umständen sogar um ein sich noch in der Schreiberschule befindliches Kind. Bisher sind Heilmittel gegen Weinen und Angst hauptsächlich für Kinder belegt, denen Amulette gegen die Furcht gefertigt werden. In vielen Fällen werden dazu bestimmte Psalmenverse niedergeschrieben, siehe dazu etwa die spät-byzantinischen Heilmittel gegen das Weinen bei Kindern in A.

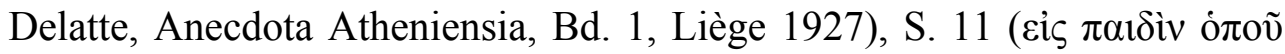

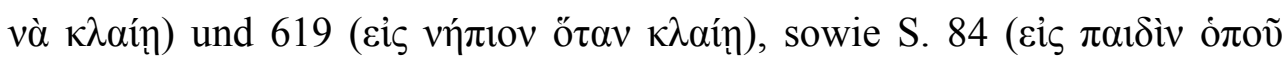

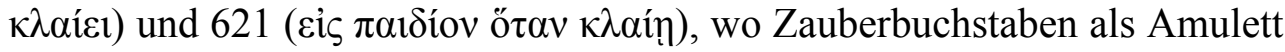
auf Papier bzw. auf Lorbeerblätter geschrieben und auf dem Kissen der Patienten platziert werden sollen oder aufgelöst als Trunk eingenommen werden (Hinweis von Michael Zellmann-Rohrer). In einem Papierkodex des 11. Jh.s der Kairoer Geniza findet sich die Anweisung, bei einem weinenden Kind den Psalm 9 auf Pergament zu schreiben und als Amulett zu tragen, siehe Schäfer und Shaked, Magische Texte aus der Kairoer Geniza, Bd. 1, text T-S K 1.28, f. 3a.1-3 (Hinweis ebenfalls von Michael ZellmannRohrer). Auch gegen die Angst bei Kindern werden in späten byzantinischen Rezepten bestimmte Psalmenverse vorgeschlagen, wie etwa im Wiener Kodexfragment ÖNB cod. hist. gr. 129 f. 12r-v, § 39, hier aus der Neuedition von Michael Zellmann-Rohrer (in Vorbereitung) zitiert: ó $\rho \kappa \zeta^{\prime} \kappa \alpha \grave{~ o ́ ~}$

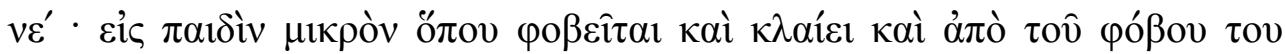

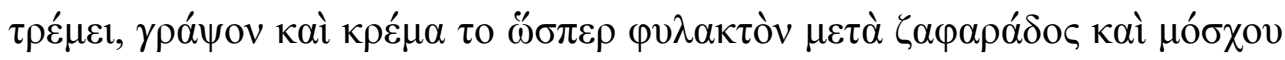

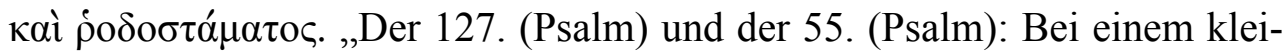
nen Kind, das sich fürchtet und weint und vor Angst zittert: Schreibe (die Psalmen) und hänge es wie ein Amulett mit Safran, Moschus und Rosenöl.“ Angstzustände und Schlaflosigkeit bei Erwachsenen scheinen ganz ähnlich

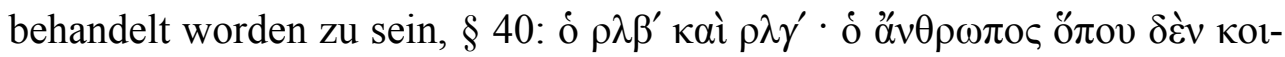

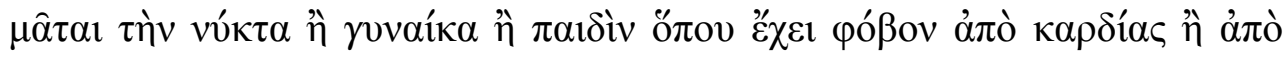

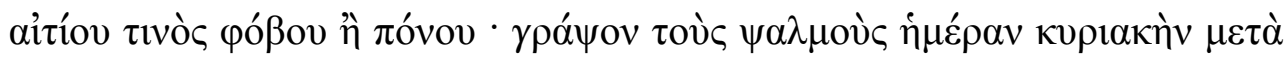




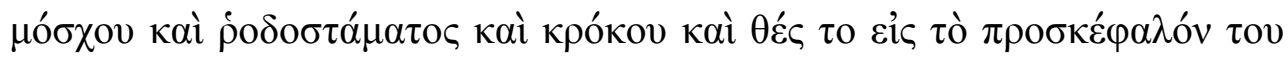

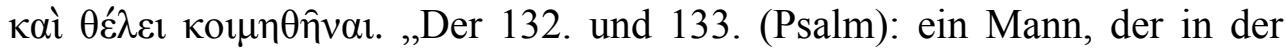
Nacht nicht schläft - oder eine Frau, oder ein Kind -, der Angst hat, innerlich oder verursacht durch irgendeine Sorge oder einen Schmerz: Schreibe die Psalmen am Sonntag mit Moschus, Rosenöl und Safran; lege es auf sein Kissen und er wird schlafen."

6 rechts: Hp- hier für $\mathrm{\epsilon p}$ - bzw. $\overline{\mathrm{p}}-$.

15 rechts: Am Zeilenende ist das letzte Alpha des Wortes manid aus Platzgründen über der Zeile geschrieben worden. Der Begriff $\mu \alpha v i ́ \alpha$ scheint bislang weder in griechischen noch in koptischen Heilmittelsammlungen und Rezepten in Erscheinug getreten $\mathrm{zu}$ sein. Als ein geistiges Leiden aufgrund mentaler Verirrung, das im Zusammenhang mit Bildung und Gelehrsamkeit, wie sie etwa bei einem Berufsschreiber oder Beamten anzunehmen wäre, auftreten kann, findet sich $\mu \alpha v i ́ \alpha$ aber z.B. in der

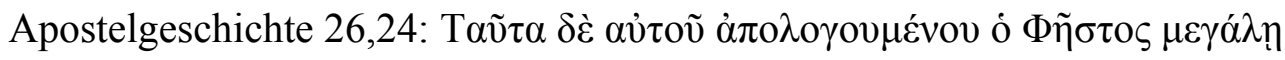

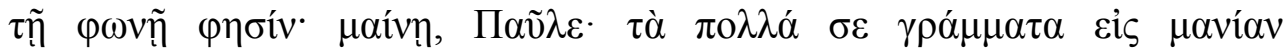
$\pi \varepsilon \rho \iota \rho \varepsilon ́ \pi \varepsilon 1$. „Als er (Paulus) aber dies zu seiner Verteidigung vorbrachte, sprach Festus mit lauter Stimme: ,Paulus, du bist von Sinnen! Das viele Studieren bringt dich um den Verstand! ““ (Übers. Schlachter, 2000)

Auch in P.Lond.Copt. I 344, der koptischen Märtyrerlegende des Mönchs Pamoun und seines Bruders Sarmata, (8./9. Jh.), entrüstet sich der Hegemon Armenios über die beiden Mönche, die nicht auf seine Züchtigungen reagieren, sondern stattdessen weiter mit scheinbar gelehrten Argumen-

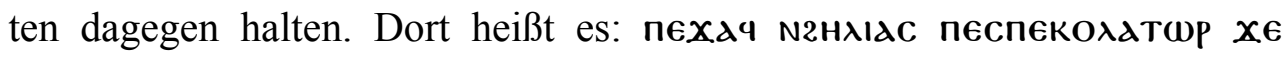
MIINAY GMANIA ENG2 NOE NMMONaXOC CNAY NTE KHME „Er (der Hegemon Armenios) sprach zu Elias, dem Wachmann (speculator): ,Ich habe noch nie einen solchen Wahnsinn (mania) gesehen, wie den der beiden ägyptischen Mönche! ““

In die gleiche Richtung weist die Mahnung des Hegemons Arianos an den heiligen Kollouthos, den er vergeblich zur Vernunft und zur Gesetzestreue $\mathrm{zu}$ bewegen sucht. Arianos wirft dem Märtyrer geistige Verwirrung und wahnsinnigen Hochmut vor, Fol. 89r II,30-89v I,10: MEүXI $\lambda d \lambda \gamma$

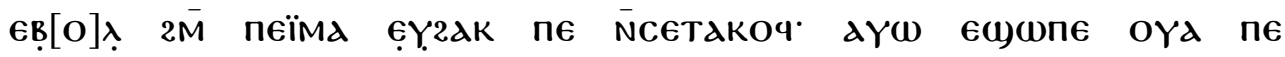

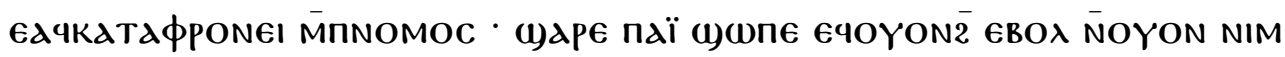

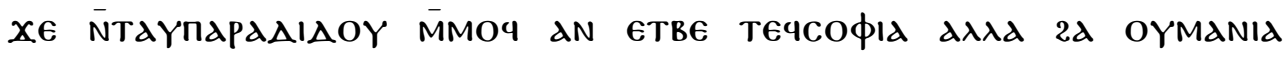
MMNT̄GoYwo• „Niemand, der vernünftig ist, wird je von hier (dem 
Gerichtssaal) fortgeführt und getötet. Wenn jemand das Gesetz missachtet hat, pflegt dieses keinem offenbar zu sein, dass das, was ihn dazu brachte, aufgrund seiner Weisheit ( бoфí $\alpha$ ) geschah, sondern durch hochmütigen

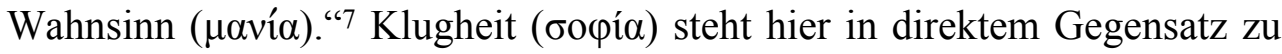
Mania.

Auch Kollouthos selbst diagnostiziert bei einem armen Bettler geistige Verirrung ( $\mu \alpha v^{\prime} \alpha$ ), als dieser behauptet, der heilige Arzt und Wunderheiler hätte gerade eben bei ihm seine zuvor verkrüppelte Hand durch bloße Berührung genesen lassen. Kollouthos verbittet sich eine solche Behauptung mit dem Verweis darauf, dass wenn überhaupt nur Christus

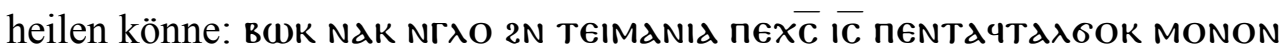
aNr оүрФмє NPEчрNoвє „Verschwinde und lass von diesem Wahnsinn ( $\left.\mu \alpha v^{\prime} \alpha\right)$ ab! Jesus Christus ist es, der dich geheilt hat. Ich bin nur ein sündiger Mensch." 8

Im hier vorliegenden Krankheitsfall könnte es sich also durchaus um die geistige Verwirrung eines angehenden Beamten handeln, die sich durch Angstzustände und Weinkrämpfe manifestierte und mittels eines Schutzamuletts bezwungen werden sollte.

14-16 links: Zu Salomons Fähigkeiten als Exorzist siehe Särkiö, Salomo und die Dämonen, 314-317, zu Amuletten und Heilmitteln in der Tradition Salomons siehe bes. 315 Anm. 49 und 50. Vielleicht läuft im Anschluß an die rhetorische Frage Salomons das Rezept gegen die Schwellung neben Zauberformeln auf den Einsatz der bekannten Heilpflanze hinaus, die auch Salomonssiegel (Polygonatum multiflorum) oder Weißwurz genannt wird und unter anderem bei verletzungsbedingten Schwellungen in der Naturmedizin zur Anwendung kommt, siehe dazu z.B. B. Baur-Müller, Westliche Heilpflanzen in der chinesischen Medizin, Berlin, Heidelberg 2016, 44, oder unter http://www.barfers.de/salomons.html. Bei W. C. Till, Die Arzneikunde der Kopten, Berlin 1951, 37-38, findet sich jedoch unter dem

7 Siehe zuletzt Schenke, Das koptisch hagiographische Dossier des Heiligen Kolluthos, $42-45$.

${ }^{8}$ S. E. Thompson, 'Encomium on St. Coluthus (M591, ff. 94r-121v), attributed to Isaac of Antinoe, in: L. Depuydt (ed.), Encomiastica from the Pierpont Morgan Library: Five Coptic Homilies Attributed to Anastasius of Euchaita, Epiphanius of Salamis, Isaac of Antinoe, Severian of Gabala, and Theopempus of Antioch, CSCO 544: Copt. 47, 47-83 (Text) und CSCO 545: Copt. 48, 37-64 (Übersetzung), Louvain 1993, Text, S. 59, Z. 19 20. 
Eintrag für ()גвє (Zeile 14) bzw. ()גчє „Geschwülste“ oder „Schwellungen“ kein Rezept mit dieser Pflanze verzeichnet.

15-16 links: Das Ypsilon in 620YN steht über der Zeile geschrieben.

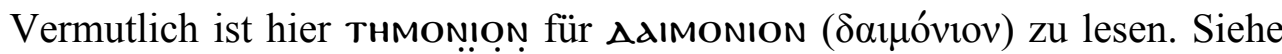
ähnliche Schreibweisen bei A. Kropp, Ausgewählte koptische Zaubertexte, Bd. I, Brüssel 1931, 90: TEMONION und TEMWNIWN.

\section{Übersetzung}

(Zeile 1-3) Bezüglich einer Frau, die blutet: Fertige diese Dinge! Binde sie (diese Dinge) an sie. Hefte sie an ihre Hüfte und an ihren Unterleib! An-

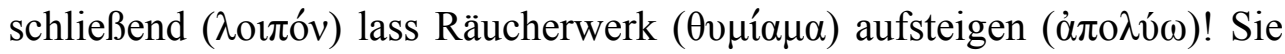
wird sich schnell ( $\tau \alpha \chi v ́)$ erholen.

(Zeile 4-12 links) REIHEN MIT ZAUBERBUCHSTABEN Schreibe diese Dinge mit Tinte ( $\mu \varepsilon \dot{\lambda} \alpha \alpha$ ) auf Holz (choubi) (oder) Papyrus ( $\chi \alpha ́ \rho \tau \eta \varsigma)$. Hefte sie (diese geschriebenen Dinge) an sie (die blutende Frau)! Schön ist das,

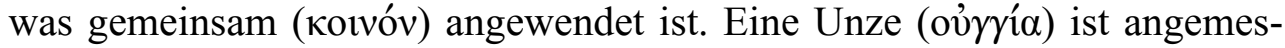

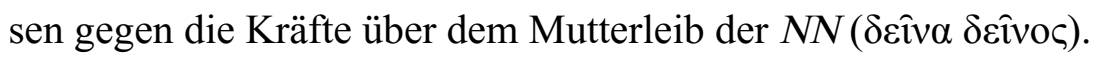

(Zeile 4-16 rechts) Ein Schutzmittel für einen jungen Beamten, der weint und sich fürchtet: REIHEN MIT ZAUBERBUCHSTABEN Befreie den Lei-

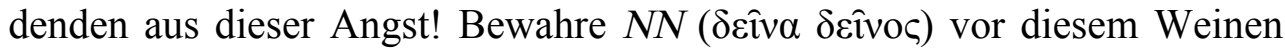
und diesem geistigen Leiden ( $\mu \alpha v i ́ \alpha)$. Komm! Komm! Schnell ( $\tau \alpha \chi u ́)$ ! Schnell ( $\tau \alpha \chi v ́) !$

(Zeile 14-16 links) Bezüglich eines Mannes, der anschwillt: Der König

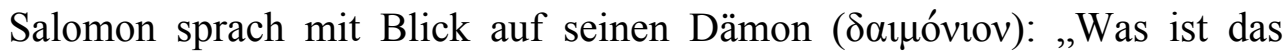
Heilmittel gegen diesen?" [...]

Gesa Schenke 